1999. Interphase FISH for rapid identification of a Down syndrome animal model. Cytogenet. Cell Genet. 86:285-287.

9. Korenberg, J.R., X.N. Chen, K.L. Devon, D. Noya, M.L. Oster-Granite, and B.W. Birren. 1999. Mouse molecular cytogenetic resource: 157 BACs link the chromosomal and genetic maps. Genome Res. 9:514-523.

10. Truett, G.E., P. Heeger, R.L. Mynatt, A.A. Truett, J.A. Walker, and M.L. Warman. 2000. Preparation of PCR-quality mouse genomic DNA with hot sodium hydroxide and tris (HotSHOT). BioTechniques 29:52-54.

11. Hartigan, J.A. and M.A. Wong. 1979. A K-means clustering algorithm. Appl. Stat. 28: 100-108.

12. Tesson, L., J.M. Heslan, S. Menoret, and I. Anegon. 2002. Rapid and accurate determination of zygosity in transgenic animals by real-time quantitative PCR. Transgenic Res. 11:43-48.

Received 8 May 2003; accepted 22 October 2003.

Address correspondence to Muriel T. Davisson, The Jackson Laboratory, 600 Main Street, Bar Harbor, ME 04609, USA. e-mail:mtd@jax.org

\title{
Annealing control primer system for improving specificity of PCR amplification
}

\author{
In-Taek Hwang, Yun-Jee Kim, Seung-Hyun Kim, Chae-II Kwak, \\ Young-Yun Gu, and Jong-Yoon Chun \\ Seegene Life Science Laboratory, Seoul, South Korea
}

BioTechniques 35:1180-1184 (December 2003)

A novel primer designed to improve the specificity of PCR amplification, called the annealing control primer (ACP), comprises a tripartite structure with a polydeoxyinosine [poly $(d I)]$ linker between the $3^{\prime}$ end target core sequence and the $5^{\prime}$ end nontarget universal sequence. We show that this ACP linker prevents annealing of the $5^{\prime}$ end nontarget sequence to the template and facilitates primer hybridization at the $3^{\prime}$ end to the target sequence at specific temperatures, resulting in a dramatic improvement of annealing specificity. The effect of this linker is demonstrated by the incorporation of ACP sequences as primers during the amplification of target nucleotide sequence and as hybridization probes in the genotyping of single nucleotide polymorphisms. This is the first report to show that a poly(dI) linker between two different sequences of ACP forms a bubble-like structure and disrupts or destabilizes DNA duplex formation at certain annealing temperatures.

\section{INTRODUCTION}

The success of PCR amplification relies solely on the specificity with which a primer anneals to its target sequences. Therefore, it is important to optimize this molecular interaction (1). The annealing temperature is critical for determining whether a primer binds only to its perfect complement or to sequences with one or more mismatches. By adjusting the annealing temperature, one can alter the specificity of pairing between template and primer. Numerous approaches have been introduced to increase primer annealing specificity. Longer primers with universal, homopolymer, or loop sequence tails at their $5^{\prime}$ ends improve the specificity of PCR amplification and the stability of hybridization (2-4). However, this approach does not abrogate nonspecific hybridization resulting from the involvement of the tail or loop sequence in the priming reaction. This limitation of longer primers necessitates the specification of the annealing sequences of the primers to exclude nonspecific annealing. Here we describe a novel annealing control primer (ACP) that specifically targets sequence hybridization to the template via a polydeoxyinosine [poly $(\mathrm{dI})]$ linker. The structure of ACP comprises (i) a $3^{\prime}$ end region with a target core nucleotide sequence that substantially complements the template nucleic acid for hybridization; (ii) a $5^{\prime}$ end region with a nontarget universal nucleotide sequence; and (iii) a poly(dI) linker bridging the $3^{\prime}$ and $5^{\prime}$ end sequences.

\section{Rationale of Annealing Control Primer Oligonucleotides}

The presence of universal bases in a primer, such as deoxyinosine $(5,6)$, 1 -( $2^{\prime}$-deoxy- $\beta$-D-ribofuranosyl)-3-nitropyrrole (7), and 5-nitroindole (8), leads to low annealing temperatures due to weaker hydrogen bonding interaction in base pairing. In this study, we show that the presence of a poly(dI) linker between the $3^{\prime}$ end and $5^{\prime}$ end sequences of a primer generates a region with a lower melting temperature $\left(\mathrm{T}_{\mathrm{m}}\right)$ via the formation of a bubble-like structure at specific temperatures. The poly(dI) linker in the bubble-like structure affects the annealing of each region of the primer. Essentially, the ACP linker prevents the $5^{\prime}$ region from annealing under conditions in which the target $3^{\prime}$ sequence anneals to the template at the original annealing temperature. 


\section{MATERIALS AND METHODS}

\section{First-Strand cDNA Synthesis}

Total RNAs (Seegene, Seoul, Korea) from 18.5-day-old mouse conceptus tissues were isolated and used for the synthesis of first-strand cDNA by reverse transcriptase as previously described (9). The cDNA synthesis primer sequence was dT-ACP1 (5'CGTGAATGCTGCGACTACGATIIIIITTTTTTTTTTTTTTTTTT- $3^{\prime}$ ), in which the poly $(\mathrm{dT})_{20}$ at the $3^{\prime}$ end was replaced as poly $(\mathrm{dT})_{18}$.

\section{Amplification of a RIKEN cDNA Target Sequence}

First-strand cDNA was synthesized from mouse conceptus total RNA [18.5 days postcoitus (dpc)] using dT-ACP1 as specified above. A 635-bp fragment of RIKEN full-length cDNA (accession no. NM 027815) (RIKEN, Saitama, Japan) was amplified using conventional primers or ACP (Table 1). A two-stage amplification reaction was conducted in $50 \mu \mathrm{L}$ reaction mixture comprising 50 ng first-strand cDNA, $5 \mu \mathrm{L} 10 \times$ buffer (Promega, Madison, WI, USA), $5 \mu \mathrm{L}$ $25 \mathrm{mM} \mathrm{MgCl}, 5 \mu \mathrm{L}$ dNTP mixture (2 $\mathrm{mM}$ each), $1 \mu \mathrm{L} 5^{\prime}$ primer $(10 \mu \mathrm{M}), 1$ $\mu \mathrm{L} 3^{\prime}$ primer $(10 \mu \mathrm{M})$, and $0.5 \mu \mathrm{L} \mathrm{Taq}$ DNA polymerase $(5 \mathrm{U} / \mu \mathrm{L})$ (Promega). We used the following PCR conditions: two cycles of $94^{\circ}$ for $1 \mathrm{~min}, 50^{\circ}$ for 3 min, and $72^{\circ} \mathrm{C}$ for $1 \mathrm{~min}$; followed by 40 cycles of $94^{\circ}$ for $40 \mathrm{~s}, 65^{\circ}$ for $40 \mathrm{~s}, 72^{\circ} \mathrm{C}$ for $40 \mathrm{~s}$, and a 5-min final extension cycle at $72^{\circ} \mathrm{C}$. The amplified products were observed on $2 \%$ agarose gel.

\section{Amplification of a Short Fragment Containing a p53 Single Nucleotide Polymorphism}

A 349-bp fragment containing a single nucleotide polymorphism (SNP) in exon 4 of the human p53 gene was amplified using human genomic DNA templates and the following primers, P53N-ACP (5'TATGAATGCTGTGACGCCGAIIIIICCTCTGACTGCTCTTTTCAC-3') and P53C-ACP (5'-TCACAGAAGTATGCCAAGCGAIIIIIATTGAAGTCTCATGGAAGCC-3'). DNA templates were obtained from human blood samples containing an SNP of mutant ho- mozygotes in exon 4 of TP53 (the tumor protein p53 gene). PCR was performed in a $50-\mu \mathrm{L}$ reaction mixture comprising $50 \mathrm{ng}$ genomic DNA, $5 \mu \mathrm{L} 10 \times$ PCR buffer, $5 \mu \mathrm{L} 25 \mathrm{mM} \mathrm{MgCl}{ }_{2}, 5 \mu \mathrm{L}$ dNTP mixture ( $2 \mathrm{mM}$ each), $1 \mu \mathrm{L}$ P53N-ACP $(10 \mu \mathrm{M}), 1 \mu \mathrm{L}$ P53C-ACP $(10 \mu \mathrm{M})$, and $0.5 \mu \mathrm{L}$ Taq DNA polymerase $(5 \mathrm{U} / \mu \mathrm{L})$. The PCR conditions used were 30 cycles of $94^{\circ}$ for $40 \mathrm{~s}, 65^{\circ}$ for $40 \mathrm{~s}$, and $72^{\circ} \mathrm{C}$ for $40 \mathrm{~s}$, followed by a 5 -min final extension cycle at $72^{\circ} \mathrm{C}$. Amplified products were purified using a QIAquick ${ }^{\circledR}$ PCR purification kit (Qiagen, Valencia, CA, USA) and employed as probes in allele-specific dot blot hybridization.

\section{Allele-Specific Dot Blot Hybridization}

Oligonucleotides $(6 \mu \mathrm{L}$ of $100 \mu \mathrm{M})$ were spotted on a Hybond ${ }^{\mathrm{TM}} \mathrm{N}^{+}$membrane (Amersham Biosciences, Piscataway, NJ, USA) and immobilized using an optimized UV cross-linking procedure. Initial hybridization was conducted using the radioactively labeled 349-bp fragment in $10 \mathrm{~mL}$ QuikHyb $^{\circledR}$ solution (Stratagene, La Jolla, CA, USA) at $40^{\circ} \mathrm{C}$ for $2 \mathrm{~h}$. The hybrid was washed $3-5$ times with a buffer solution of $2 \times$ standard saline citrate (SSC) and $1 \times \mathrm{SSC} / 0.1 \%$ sodium dodecyl sulfate (SDS) at room temperature for 1-10 $\mathrm{min}$ and exposed to film at $-80^{\circ} \mathrm{C}$. The resultant membrane filter used in the first step was re-employed for a second hybridization without stripping to remove the probe used in the first hybridization. The second hybridization step was conducted using the first hybridized filter and $\left[\gamma^{32} \mathrm{P}\right]$-labeled oligonucleotide comprising a sequence complementary to the second hybridization region of longer conventional or ACP oligonucleotides in $10 \mathrm{~mL}$ QuikHyb solution at $58^{\circ} \mathrm{C}$ for $2 \mathrm{~h}$. The hybrid was washed three times with a buffer solution of $2 \times \mathrm{SSC}$ and $1 \times \mathrm{SSC} / 0.1 \% \mathrm{SDS}$ at $60^{\circ} \mathrm{C}$ for $5 \mathrm{~min}$ and exposed to film at $80^{\circ} \mathrm{C}$. The radiation dose of each dot was measured by autoradiography to evaluate the strength of the hybridization.

\section{RESULTS AND DISCUSSION}

To determine whether the poly(dI) linker of ACP prevents annealing of the $5^{\prime}$ region under conditions in which the $3^{\prime}$ region is fully hybridized to the template 
Table 1. Oligonucleotides Used in this Study

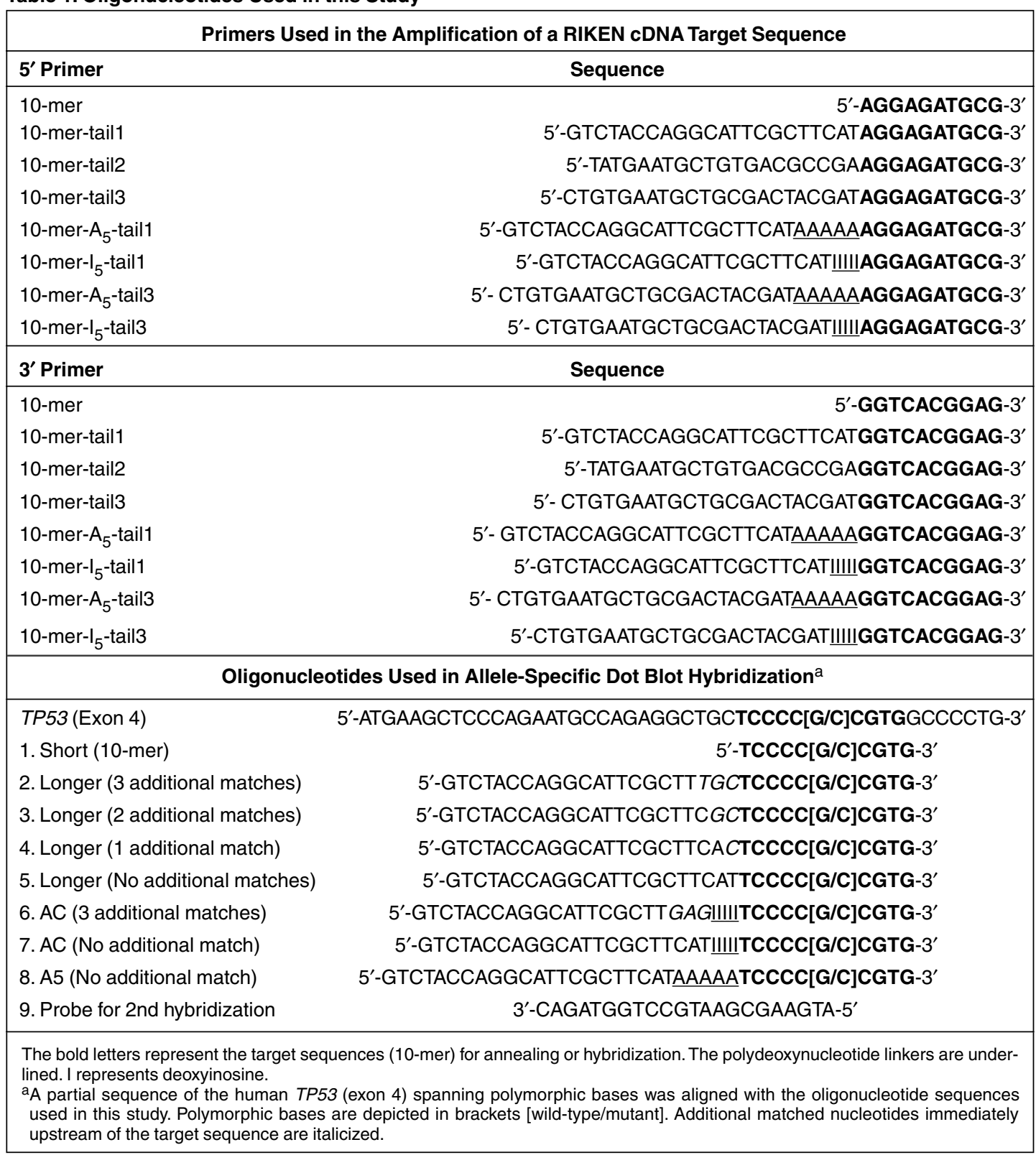

products, regardless of the nontarget universal sequences at the $5^{\prime}$ regions (Figure 1 , lanes 4, 8, and 9).

Three major conclusions can be drawn on the basis of these results. (i) Nontarget universal sequences of longer conventional primers are involved in primer annealing, which results in numerous nonspecific products. (ii) Nontarget universal sequences of $\mathrm{ACP}$ are not involved in annealing while the $3^{\prime}$ target regions anneal to the template during first-stage PCR, resulting in specific products only. (iii) Linkers composed of other polydeoxynucleotides [e.g., $(\mathrm{dA})_{5}$ or $(\mathrm{dT})_{5}$ ] do not have the same benefits as the poly(dI) linker. Thus, our data clearly show that the poly(dI) linker of ACP facilitates the targeting of the $3^{\prime}$ sequences to be hybridized to the template under stringent conditions. Although a two-stage PCR is applied to both ACP and longer primer systems to increase annealing

at the first annealing temperature, the amplification of a RIKEN cDNA target sequence was compared using ACP and longer conventional primers. Both ACP and longer primers comprise 10-mer target sequences at their $3^{\prime}$ ends and nontarget universal sequence tails at their $5^{\prime}$ ends (Table 1). Consistent with our expectations, a pair of longer conventional primers with the same structure as ACP [except for the presence of the poly(dI) linker] generated many nonspecific products, including the target product (Figure 1, lane 2). Upon replacing the 5 ' sequences of the conventional primers with other nontarget universal sequences, different patterns of nonspecific products were generated, as observed on a $2 \%$ agarose gel (Figure 1, lanes 3, 6, and 7). In contrast, a pair of ACP primers produced the expected target product (Figure 1 , lanes 5,10 , and 11 , confirmed by cloning and sequencing; data not shown). We further examined whether other common nucleotide residues [e.g., $(\mathrm{dA})_{5}$ or $(\mathrm{dT})_{5}$ ] would be applicable for constructing a polydeoxynucleotide linker by substituting deoxyinosine with polydeoxyadenosine $[p o l y(d A)]$. Primers with the poly(dA) linker generated nonspecific specificity, the longer primers are not free from the problems caused by the involvement of nonspecific tail or loop sequences in primer hybridization (2-4). In contrast, the ACP system utilizing the two-stage PCR prevents the nonspecific sequences from annealing to the template due to the effects of the poly(dI) linker, resulting in a significant increase in annealing specificity. This is the first report to describe the role of the poly $(\mathrm{dI})$ linker in distinguishing between two different annealing sequences of PCR primers in association with annealing temperature.

The key conditions and parameters of 
this technique for the amplification of a target sequence are as follows. The first annealing temperature should be $10^{\circ}-$ $20^{\circ} \mathrm{C}$ higher than the $\mathrm{T}_{\mathrm{m}}$ of the $3^{\prime}$ target core sequence of the ACP. The length of the $3^{\prime}$ end target sequence is designed to have a $\mathrm{T}_{\mathrm{m}}$ ranging from $30^{\circ}-55^{\circ} \mathrm{C}$, and shorter primer sequences (between 10and 15-mers) are more compatible for maximizing the advantage of the ACP system, where the GC content is dependent on the length of the $3^{\prime}$ end target sequence and its $T_{m}$. Further, the other PCR parameters, such as buffer and salt

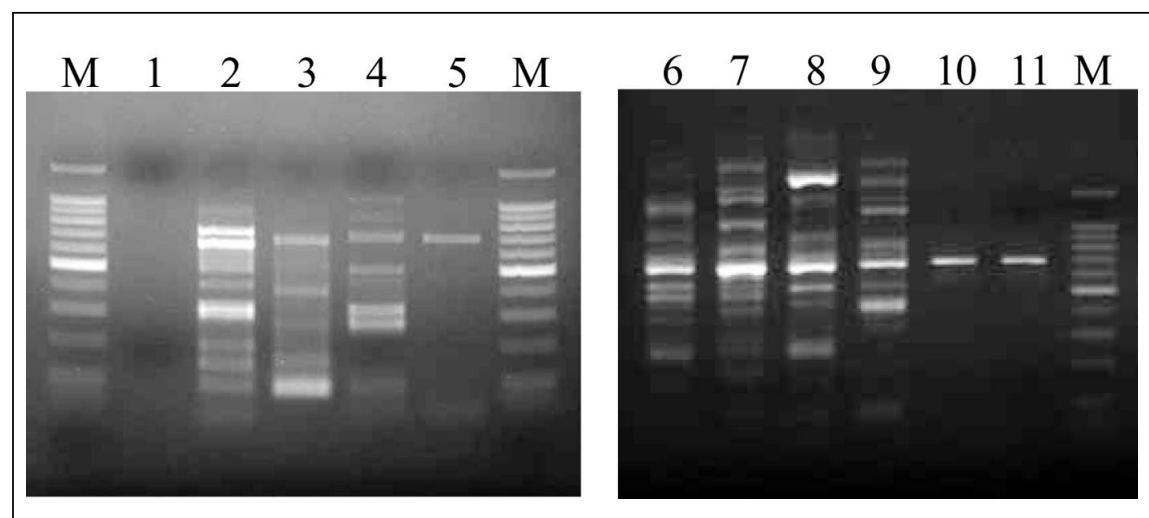

Figure 1. Amplification of a target RIKEN cDNA fragment using conventional primers or annealing control primer. Short conventional primers (such as 10-mers) used as a negative control produced no visible products, since their melting temperatures $\left(\mathrm{T}_{\mathrm{m}} \mathrm{s}\right)$ were $32^{\circ}\left(5^{\prime}\right.$ primer) and $34^{\circ} \mathrm{C}\left(3^{\prime}\right.$ primer), but the first annealing temperature was $50^{\circ} \mathrm{C}$ (lane 1$)$. Primer combinations $\left(5^{\prime}\right.$ to $\left.3^{\prime}\right)$ are as follows: lane 1, 10-mer + 10-mer; lane 2, 10-mer-tail1 + 10-mer-tail2; lane 3, 10-mer-tail2 + 10-mer-tail1; lane

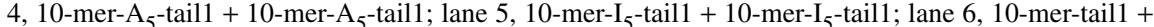
10-mer-tail3; lane 7, 10-mer-tail3 + 10-mer-tail1; lane 8, 10-mer- $\mathrm{A}_{5}$-tail1 + 10-mer- $\mathrm{A}_{5}$-tail3; lane 9, 10-

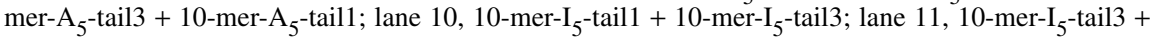
10-mer-I $\mathrm{I}_{5}$-tail1. M represents the 100-bp marker (Promega).

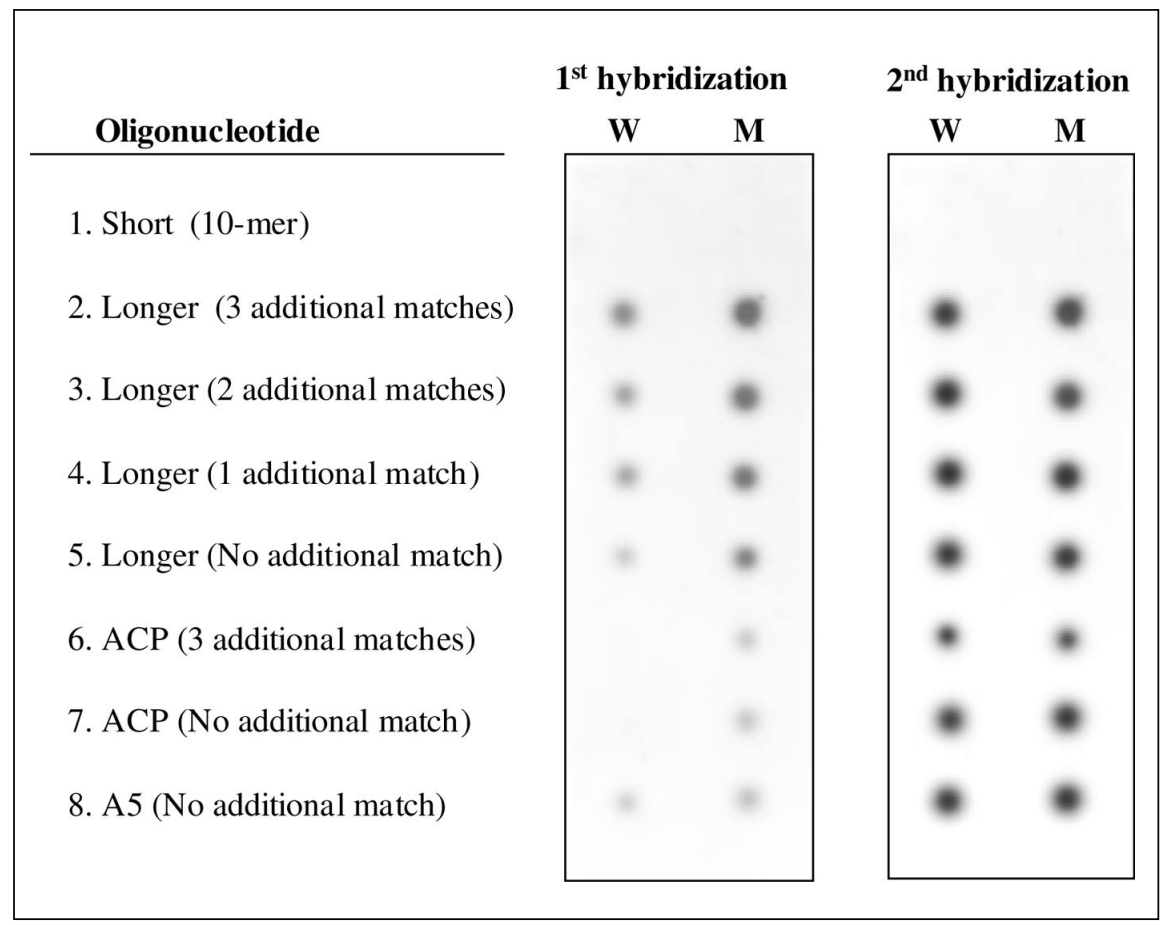

Figure 2. Allele-specific dot blot hybridization for distinguishing a single base mismatch. $\mathrm{Al}$ lele-specific short (row 1), longer (rows 2-5 and 8), and annealing control primer (ACP) (rows 6 and 7) oligonucleotides were immobilized on the membrane. Longer and ACP oligonucleotides have tail sequences at their $5^{\prime}$ end with additional matched nucleotides (0-3) immediately upstream of the target sequence as indicated. W, wild-type oligonucleotide; M, mutant-type oligonucleotide. 
concentration, depend on the DNA polymerase used in the reaction.

To determine the optimum length of the linker, ACP sequences were examined by varying the number of deoxyinosine residues in the linker. Optimum function was observed at a length of five bases (data not shown). Accordingly, the fivebase linker was used throughout the experiments described in this report.

The utility of the poly(dI) linker of ACP in PCR applications was additionally confirmed by evaluating its effects on a single base mismatch. We compared the discriminatory effects of ACP and conventional oligonucleotides on SNPs. Oligonucleotides were immobilized on a nylon membrane. A region containing an SNP in exon 4 of TP53 was amplified using ACP. This polymorphism (10) is expressed as an Arg to Pro substitution at position 72 (as a result of replacing $\mathrm{G}$ with $\mathrm{C}$ ). A 349-bp sequence of the TP53 gene was amplified from mutant homozygotes.

Surprisingly, the 10-bp $3^{\prime}$ target sequence of allele-specific ACP oligonucleotides effectively discriminated between perfectly matched and one base mismatched duplexes (Figure 2, rows 6 and 7). In contrast, longer allele-specific conventional oligonucleotides failed to distinguish between the two duplex types (Figure 2, rows 2-5). The poly(dA) linker did not function in the same manner as the poly(dI) linker (Figure 2, row 1), which is consistent with data from PCR applications (Figure 1). These results further support our hypothesis that the poly(dI) linker of ACP oligonucleotides specifically targets the hybridization sequence to the $3^{\prime}$ allele-specific region under conditions of the first hybridization step, resulting in SNP discrimination.

Furthermore, regardless of the presence of three additional complementary nucleotides in the $5^{\prime}$ sequence, the poly(dI) linker of ACP oligonucleotides prevents this region from hybridizing to the template (Figure 2, row 6). The same phenomenon is evident in ACPbased PCR amplification.

Shorter, conventional oligonucleotides did not form hybrids under such high-stringency conditions (Figure 1, row 1). In contrast, $\mathrm{ACP}$ and longer conventional oligonucleotides formed hybrids with mutant genomic DNA fragments under these conditions (Fig- ure 2, rows 2-8). Our results imply that the additional tailing sequences of both longer conventional and ACP oligonucleotides increase the strength (i.e., efficiency) of the first hybridization region, thus allowing higher hybridization and washing temperatures. Our results are consistent with a spacer effect proposed by Saiki and co-workers (3).

To confirm the results of the first annealing step, a second step was conducted using the resultant membrane filter employed in the first step without stripping and a labeled oligonucleotide probe complementary to the nontarget universal sequence of ACP primers. The results of the second hybridization step showed that each spot had almost equal radioactivity (Figure 2). These data suggest that the spotting and immobilization of the oligonucleotide probes were conducted equally in the membrane and that allele-specific ACP primers function effectively as probes for the discrimination of a single base mismatch in the first hybridization stage. Accordingly, the quality of the first hybridization can be verified through the second step.

Interestingly, the poly(dI) linker of ACP oligonucleotides affected not only the first hybridization sequence $\left(3^{\prime}\right.$ region) but also the second hybridization sequence $\left(5^{\prime}\right.$ region). Longer conventional oligonucleotides were not sufficiently sensitive to detect mismatched bases in the second hybridization sequence and thus failed to distinguish between a perfect match and a three-base mismatch (Figure 2). However, ACP oligonucleotides displayed a much weaker signal for mismatched bases in hybridization sequences than for a perfect match (Figure 2). These results suggest that the poly(dI) linker induces hybridization specificity for both $3^{\prime}$ and $5^{\prime}$ sequences of ACP oligonucleotides. An additional benefit of the structure of ACP oligonucleotides is that a rational universal sequence applicable to multiple templates or genes can be designed because the poly(dI) linker allows for the second sequence to hybridize separately from the first sequence, which is extremely difficult with longer conventional oligonucleotides.

In conclusion, the evaluation of ACP oligonucleotides as primers or probes in the amplification of target nucleotide sequences and SNP discrimination reveals the potential of tripartite structure oligonucleotides to increase the hybridization strength of target sequences in all fields of nucleic acid amplification and hybridization-based methods.

\section{ACKNOWLEDGMENTS}

We thank J.-Y. Lee for providing SNP samples.

\section{REFERENCES}

1.McPherson, M.J. and S.G. Møller. 2000. Optimization of PCR, p. 67-87. In PCR. BIOS Scientific Publishers, Oxfordshire, UK.

2.Brownie, J., S. Shawcross, J. Theaker, D. Whitcombe, R. Ferrie, C. Newton, and S. Little. 1997. The elimination of primer-dimer accumulation in PCR. Nucleic Acids Res. 25: 3235-3241.

3.Saiki, R.K., P.S. Walsh, C.H. Levenson, and H.A. Erlich. 1989. Genetic analysis of amplified DNA with immobilized sequence-specific oligonucleotide probes. Proc. Natl. Acad. Sci. USA 86:6230-6234.

4.Ailenberg M. and M. Silverman. 2000. Controlled hot start and improved specificity in carrying out PCR utilizing touch-up and loop incorporated primers (TULIPS). BioTechniques 29:1018-1024.

5.Martin, F.H., M.M. Castro, F. Aboulela, and I. Tinoco, Jr. 1985. Base pairing involving deoxyinosine: implication for probe design. Nucleic Acids Res. 13:8927-8938.

6.Ohtsuka, E., S. Matsuka, M. Ikehara, Y. Takahashi, and K. Matsubara. 1985. An alternative approach to deoxyoligonucleotides as hybridization probes by insertion of deoxyinosine at ambiguous codon positions. J. Biol. Chem. 260: 2605-2608.

7.Nichols, R., P.C. Andrews, P. Ahang, and D.E. Bergstrom. 1994. A universal nucleoside for use at ambiguous sites in DNA primers. Nature 369: 492-493.

8.Loakes, D. and D.M. Brown. 1994. 5-Nitroindole as a universal base analog. Nucleic Acids Res. 22:4039-4043.

9.Hwang, I.T., Y.H. Lee, B.C. Moon, K.Y. Ahn, S.W. Lee, and J.Y. Chun. 2000. Identification and characterization of a new member of the placental prolactin-like protein-C (PLP-C) subfamily, PLP-C $\beta$. Endocrinology 141:3343-3352.

10.Matlashewski, G.J., S. Tuck, D. Pim, P. Lamb, J. Schneider, J. Schneider, and L.V. Crawford. 1987. Primary structure polymorphism at amino acid residue 72 of human p53. Mol. Cell Biol. 7: 961-963.

Received 17 July 2003; accepted 3 October 2003.

Address correspondence to Jong-Yoon Chun, Seegene Life Science Laboratory, 142-21 Samsung-dong, Kangnam-gu, Seoul 135-090, South Korea.e-mail:chun@see-gene.com 